\title{
Voriconazole is safe and effective as prophylaxis for early and late fungal infections following allogeneic hematopoietic stem cell transplantation
}

T. Martin, M. Sharma, L. Damon, L. Kaplan, B.J. Guglielmo, M.Working, R. O'Malley, J. Hwang, C. Linker. Voriconazole is safe and effective as prophylaxis for early and late fungal infections following allogeneic hematopoietic stem cell transplantation.

Transpl Infect Dis 2010: 12: 45-50. All rights reserved

\begin{abstract}
Seventy-two patients undergoing allogeneic transplantation were treated with voriconazole (VOR) as antifungal prophylaxis starting from day -2 of transplantation and continuing until withdrawal of immunosuppression. Patients were assessed for safety and the incidence of definite, probable, or possible fungal infection throughout transplantation was evaluated.VOR was well tolerated. Only $14 \%$ of patients required interruption of VOR therapy because of toxicity: liver toxicity $(8 \%)$, cardiac $Q-$ T interval prolongation $(1 \%)$, or other side effects $(5 \%)$. In the early posttransplant period ( $<120$ days), only 2 patients developed invasive fungal infection: 1 mucormycosis infection and 1 disseminated Aspergillus infection. In the late post-transplant period ( $>120$ days), no patients developed probable or definite fungal infection while receiving VOR. No Candida infections were seen in either period. These data suggest that fungal prophylaxis with VOR following allogeneic transplantation is safe and effective.
\end{abstract}

\author{
T. Martin ${ }^{1}$, M. Sharma ${ }^{2}$, L. Damon', \\ L. Kaplan ${ }^{1}$, B.J. Guglielmo ${ }^{3}$, M. Working ${ }^{4}$, \\ R. O'Malley ${ }^{4}$, J. Hwang ${ }^{5}$, C. Linker ${ }^{1}$ \\ ${ }^{1}$ Division of Hematology/Oncology, University of California- \\ San Francisco, San Francisco, California, USA, ${ }^{2}$ Division of \\ Hematology/Oncology, University of Chicago, Chicago, \\ Illinois, USA, ${ }^{3}$ Department of Clinical Pharmacy, School of \\ Pharmacy, University of California-San Francisco, San \\ Francisco, California, USA, ${ }^{4}$ Department of Medicine, \\ University of Michigan Medical Center, Ann Arbor, Michigan, \\ USA, ${ }^{5}$ Biostatistics Core Facility, University of California- \\ San Francisco, San Francisco, California, USA
}

\author{
Key words: voriconazole; mucormycosis; Aspergillus; \\ allogeneic; stem cell transplant; fungal infections

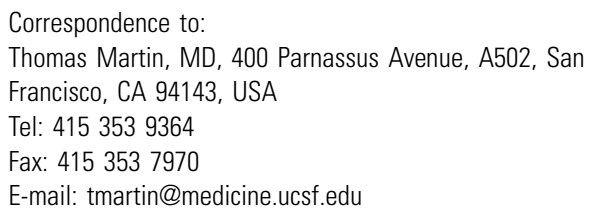

Received 12 February 2009, revised 1 May 2009, accepted for publication 22 June 2009

DOI: 10.1111/j.1399-3062.2009.00455.x

Transpl Infect Dis 2010: 12: $45-50$
Invasive fungal infection (IFI) remains a significant cause of morbidity and mortality following allogeneic hematopoietic stem cell transplantation (alloSCT). Fluconazole (FLU) is widely used as antifungal prophylaxis in patients undergoing alloSCT. In randomized trials, FLU prophylaxis has decreased the incidence of IFIs and reduced the need for amphotericin therapy (1-3). In addition, long-term data have demonstrated a survival advantage for patients receiving FLU versus placebo following bone marrow transplant (3). However, FLU has a narrow antifungal spectrum and resistant Candida and filamentous mold infections remain problematic. In a recent randomized trial, posaconazole, a second-generation triazole with broadspectrum activity against yeasts and molds, has demon- strated improved efficacy over FLU as prophylaxis for invasive Aspergillus infection in patients with severe graftversus-host disease (GVHD) following alloSCT (4). Limitations of posaconazole include the lack of an intravenous (IV) formulation and poor bioavailability. Voriconazole (VOR) is another second-generation triazole with improved activity against IFIs including non-albicans Candida and Aspergillus species (5).VOR is available in both oral and IV formulations, has excellent bioavailability, and consequently is an attractive alternative to FLU and posaconazole for prophylaxis following alloSCT.

Since approval by the US Food and Drug Administration in 2001, VOR has been used as standard antifungal prophylaxis for patients undergoing alloSCT at the University of 
California, San Francisco (UCSF) Medical Center. In this retrospective review, we report the incidence of early and late IFIs and assess the toxicity of VOR when used as prophylaxis for alloSCT. All patients have been followed for $>1$ year after alloSCT, with a mean follow-up of 1.83 years.

\section{Material and methods}

\section{Patient selection}

The study design was a retrospective chart review of consecutive patients undergoing alloSCT at UCSF Medical Center from May 2002 through June 2004. Patients received VOR as antifungal prophylaxis and the frequency of IFI was determined. Seventy-two patients were included in this analysis and no patients were excluded due to early mortality (death within 30 days of alloSCT). All patients signed informed consent for the transplant procedure and approval was obtained for this retrospective analysis from the UCSF institutional review board.

\section{Transplantation and supportive care}

Both myeloablative and non-myeloablative conditioning regimens were utilized. Myeloablative therapy consisted of IV busulfan $(12.8 \mathrm{mg} / \mathrm{kg})$ or total body irradiation (1200 cGy) with fludarabine $\left(150 \mathrm{mg} / \mathrm{m}^{2}\right)$ or cyclophosphamide $(120 \mathrm{mg} / \mathrm{kg})$. Non-myeloablative therapy utilized IV busulfan at a lower dose (4.8-6.4 mg/kg), or melphalan $\left(100 \mathrm{mg} / \mathrm{m}^{2}\right)$ with fludarabine. Anti-thymocyte globulin was added for non-myeloablative alloSCT with unrelated donors. In general, GVHD prophylaxis consisted of tacrolimus (TAC) starting from day -2 with a target level of $5-15 \mathrm{ng} / \mathrm{mL}$ and methotrexate $\left(5 \mathrm{mg} / \mathrm{m}^{2}\right.$ IV once daily on days $+1,+3,+6$, and +11 ). Patients receiving nonmyeloablative unrelated alloSCTalso received mycophenolate mofetil ( $15 \mathrm{mg} / \mathrm{kg}$ twice daily [b.i.d.]) starting from day 0 and continuing until day +60 . Most patients received granulocyte colony-stimulating factor (G-CSF) mobilized peripheral blood stem cells, with a target dose of $5 \times 10^{6} / \mathrm{kg}$ recipient weight. Human leukocyte antigen (HLA) typing was performed using high-resolution molecular techniques for unrelated donors and only well-matched donors were utilized (i.e., siblings were fully HLA-matched while unrelated donors were matched at $7 / 8$ or $8 / 8$ HLA-loci [A, B, C, DR] for myeloablative SCT, or 9/10, or 10/10 HLA-loci [A, B, $\mathrm{C}, \mathrm{DR}$, and DQ] for non-myeloablative SCT). Stem cell processing was performed for ABO incompatibility according to standard blood banking procedures. By convention, the day of stem cell infusion was considered day 0 of alloSCT.
Patients were hospitalized in single rooms equipped with high-efficiency particulate air (HEPA) filter systems. Infectious disease prophylaxis included oral antibacterials (levofloxacin or moxifloxacin) during neutropenia, oral or IV antivirals starting from day -2 (acyclovir), and prophylaxis for Pneumocystis jirovecii pneumonia following engraftment (i.e., trimethoprim/sulfamethoxazole or dapsone). Oral VOR $4 \mathrm{mg} / \mathrm{kg}$ b.i.d. started from day -2 and continued until immunosuppression was discontinued ( $>100$ days). Weight-based VOR dosing was chosen to limit intra-patient variability and in an attempt to improve therapeutic drug delivery. Broad-spectrum IV antibiotics were used for episodes of febrile neutropenia. Preemptive monitoring of cytomegalovirus (CMV) infection (weekly CMV blood antigen) was used for patients who were at risk for CMV reactivation. G-CSF was given to all patients starting at day +7 and continued until absolute neutrophil count $>1500 / \mu \mathrm{L}$ for 2 consecutive days. Corticosteroids were used as first-line therapy for acute and chronic GVHD.

\section{Evaluations and definitions}

The primary objective of the study was to evaluate the incidence of early and late IFI using VOR as prophylaxis for alloSCT. IFI was evaluated according to EORTC-defined criteria (Fig. 1) (6). Briefly, definite IFI was defined as a fungal pathogen isolated by culture or identified by histology from a known sterile site (i.e., biopsy-proven disease); probable IFI included episodes with clinical evidence of disease (abnormal chest x-ray) and positive culture from the site (e.g., bronchoalveolar lavage, sputum) or positive galactomannan assay. Possible IFI included episodes with an abnormal radiology study plus 2 or more abnormal host factors (e.g., fever, sputum, cough) but no positive cultures. Plasma VOR drug levels and galactomannan assays were not routinely performed. Early IFI was defined as that

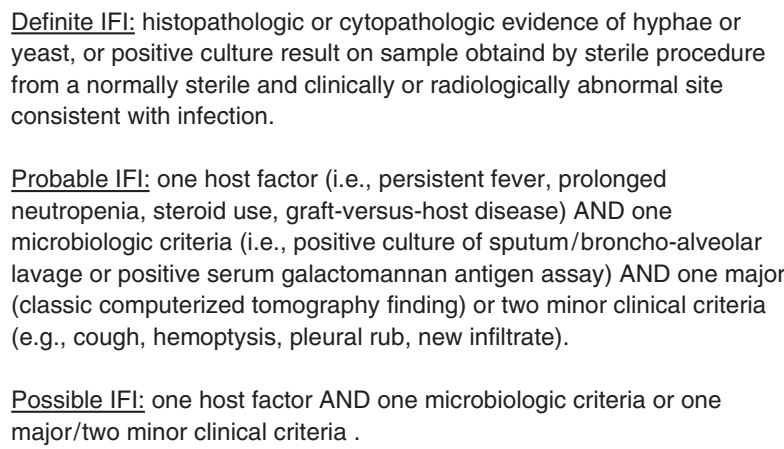

Probable IFI: one host factor (i.e., persistent fever, prolonged neutropenia, steroid use, graft-versus-host disease) AND one microbiologic criteria (i.e., positive culture of sputum/broncho-alveolar lavage or positive serum galactomannan antigen assay) AND one major (classic computerized tomography finding) or two minor clinical criteria (e.g., cough, hemoptysis, pleural rub, new infiltrate).

Possible IFI: one host factor AND one microbiologic criteria or one major/two minor clinical criteria

Fig. 1. EORTC/NIAID criteria for possible, probable, and definite invasive fungal infections (IFIs) (6). 
occurring within 120 days of alloSCT, with late IFI occurring after day 120 . All microbiologic and radiographic data for each patient were reviewed.

Secondary outcomes assessed were incidence of non-fungal infections, incidence of acute/chronic GVHD, VOR-related toxicity, treatment-related mortality, disease-free survival, and overall survival. Treatment-related mortality was defined as any non-disease-associated death occurring within 120 days of alloSCT.

\section{Results}

Subjects had a wide variety of malignant disease (Table 1). Approximately half of the patients received non-myeloabla-

\begin{tabular}{|c|c|}
\hline \multicolumn{2}{|l|}{ Patient characteristics $(n=72)$} \\
\hline Mean age at transplant in years (range) & $47(18-68)$ \\
\hline \multicolumn{2}{|l|}{ Gender } \\
\hline Female & 30 \\
\hline Male & 42 \\
\hline \multicolumn{2}{|l|}{ Ethnicity } \\
\hline White & 52 \\
\hline Asian & 10 \\
\hline Hispanic & 6 \\
\hline Black & 4 \\
\hline \multicolumn{2}{|l|}{ Diagnosis } \\
\hline Non-Hodgkin’s lymphoma & 17 \\
\hline Acute myelogenous leukemia & 16 \\
\hline Acute lymphocytic leukemia & 9 \\
\hline Myeloma/plasmacytoma & 8 \\
\hline Myelodysplasia & 7 \\
\hline Chronic myelogenous leukemia & 5 \\
\hline Chronic lymphocytic leukemia & 4 \\
\hline Hodgkin's disease & 3 \\
\hline Myeloproliferative disorders & 2 \\
\hline Metastatic prostate cancer & 1 \\
\hline \multicolumn{2}{|l|}{ Prep } \\
\hline Non-myeloablative & 41 \\
\hline Myeloablative & 31 \\
\hline \multicolumn{2}{|l|}{ Stem cell } \\
\hline Matched sib (PBSC/BM) & $37(36 / 1)$ \\
\hline Matched unrelated (PBSC/BM) & $35(24 / 11)$ \\
\hline
\end{tabular}

\section{Table 1}

Incidence of definite, probable, and possible invasive fungal infections

\begin{tabular}{|c|c|c|}
\hline & $\begin{array}{l}\text { Early ( }<120 \text { days } \\
\text { post transplant) }\end{array}$ & $\begin{array}{l}\text { Late ( }>120 \text { days } \\
\text { post transplant) }\end{array}$ \\
\hline Definite (\%) & 2 patients $(3 \%)$ & 2 patients $(3 \%)$ \\
\hline Probable (\%) & 0 patients $(0 \%)$ & 4 patients $(6 \%)$ \\
\hline Possible (\%) & 6 patients $(8 \%)$ & 6 patients ( $9 \%)$ \\
\hline
\end{tabular}

Table 2

tive conditioning and a slight majority of patients received stem cells from sibling donors. In general, most patients were considered high-risk because of adverse cytogenetics, prior disease relapse, or failure of prior autologous transplantation. The mean follow-up for survivors is 668 days (1.83 years, range $0.10-4.23$ years) after alloSCT.

\section{Primary endpoint}

Seventy-two patients were evaluated for early IFI and only 2 developed definite IFI (Table 2). One patient developed IFI following severe GVHD requiring treatment with more than 3 immunosuppressive agents. This patient was hospitalized continuously post alloSCTand died of IFI with active GVHD at approximately day +100 post alloSCT. Disseminated mucormycosis infection was identified at autopsy. The second patient developed veno-occlusive disease within 25 days following alloSCT and discontinued VOR because of elevated total bilirubin at approximately day 30 post transplant. This patient subsequently received an echinocandin for antifungal prophylaxis and developed IFI with Aspergillus and Scedosporium prolificans. The patient died of IFI at approximately day 80 post alloSCT.

No cases of probable IFI were identified and 6 cases of possible IFI occurred. Five of the patients with possible IFI responded to either broad-spectrum antibiotics or the addition of an echinocandin to VOR therapy. One patient died rapidly from pneumonia and culture-negative sepsis, presumably of bacterial origin. In general, all patients with possible IFI were more likely to have viral or bacterial etiologies for their infections.

Sixty-four patients survived longer than 120 days post transplantation and thus were evaluable for incidence of late IFI. Late IFI developed only in patients who had chronic GVHD, or who were on steroids for other reasons (relapse, possible autoimmune cytopenia). Two patients developed definite IFI (both Aspergillus) and both died of progressive infection. Both patients had been off VOR for several months before development of IFI (1 due to hyperbilirubinemia, 1 who was non-adherent with VOR prophylaxis despite chronic GVHD). The 4 patients experiencing late probable IFI also were already off VOR at the time of 
their fungal infection. The patients with possible late IFI had presumed non-fungal origin of their infections. No patients receiving VOR for fungal prophylaxis later than day 120 post alloSCTdeveloped probable or definitive late IFI.

Overall, no cases of invasive candidal infection occurred in either the early or late periods. One case of zygomycosis was identified.

\section{Secondary endpoints}

VOR was well tolerated. Ten patients (14\%) stopped VOR because of drug toxicity. The reasons for stopping included elevated liver function tests (LFT) in 6 patients, veno-occlusive disease in 2 patients, toxic TAC level in 1 patient, and prolonged cardiac corrected Q-T interval (QTc) $(>500$ $\mathrm{msec}$ ) in 1 patient. Four patients were able to restart VOR when their LFT abnormalities improved. Of note, VOR increased serum TAC levels in all patients, necessitating a $60 \%$ reduction in standard TAC dosing. Despite a low incidence of fungal disease, bacterial and viral infections were common (Table 3). The incidence of CMV reactivation was approximately $28 \%$ and gram-positive bacteremia was $24 \%$ in the early post-transplant period. Late post-transplant gram-positive bacteremia was seen in $35 \%$ of patients and late CMV re-activation occurred in approximately $10 \%$ of patients. Clostridium difficile infections were detected in

Incidence of non-fungal infections (\%) post transplant

\begin{tabular}{|c|c|c|c|}
\hline & Infection & $\begin{array}{l}\text { Early }(<120 \text { days } \\
\text { post SCT) }\end{array}$ & $\begin{array}{l}\text { Late }(>120 \text { days } \\
\text { post SCT })\end{array}$ \\
\hline \multirow[t]{11}{*}{ Bacterial } & \multicolumn{3}{|l|}{ Type } \\
\hline & Gram + & 24 & 35 \\
\hline & GNR & 10 & 17 \\
\hline & C. diff. & 15 & 11 \\
\hline & \multicolumn{3}{|l|}{ Site } \\
\hline & Blood & 17 & 28 \\
\hline & Urine & 13 & 11 \\
\hline & GI & 12 & 9 \\
\hline & Lung & 5 & 9 \\
\hline & Other & 4 & 4 \\
\hline & Total & 45 & 59 \\
\hline \multirow[t]{5}{*}{ Viral } & HSV/VZV & 3 & 5 \\
\hline & CMV antigen & 28 & 9 \\
\hline & CMV disease & 0 & 1 \\
\hline & Resp viruses & 5 & 14 \\
\hline & Total & 36 & 29 \\
\hline Mycobacterial & & 0 & 2 \\
\hline $\begin{array}{l}\text { post SCT, post } \\
\text { GNR, gram-ne६ } \\
\text { HSV, herpes si } \\
\text { cytomegalovir }\end{array}$ & $\begin{array}{l}\text { tem cell transp } \\
\text { tive rod; C. diff. } \\
\text { plex virus; VZV, } \\
\text {; Resp, respira }\end{array}$ & $\begin{array}{l}\text { ant; Gram + , gram- } \\
\text { Clostridium difficile; } \\
\text { varicella zoster virus, } \\
\text { ory. }\end{array}$ & $\begin{array}{l}\text { ositive organism; } \\
\text { l, gastrointestinal; } \\
\text { CMV, }\end{array}$ \\
\hline
\end{tabular}

Table 3

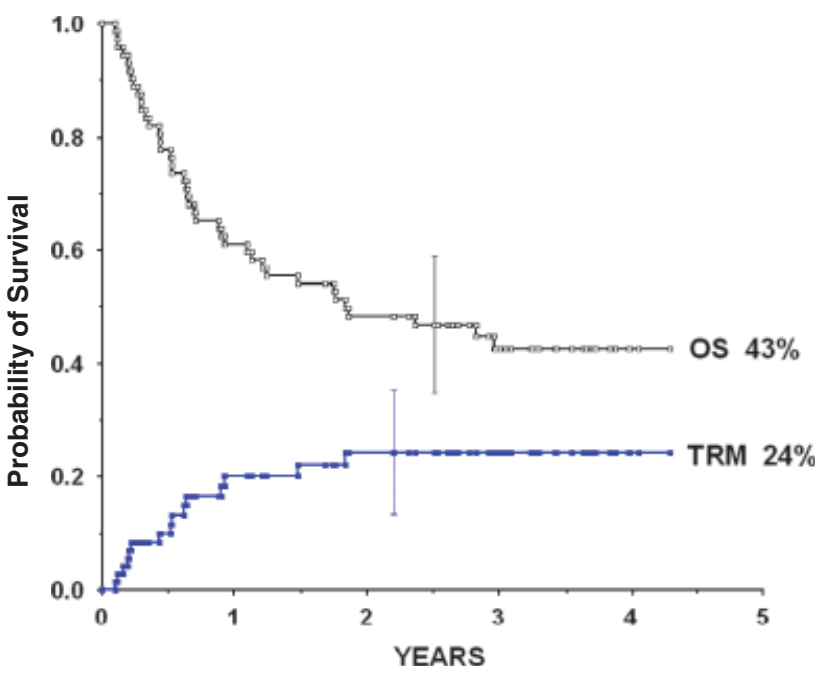

Fig. 2. Kaplan-Meier curve showing treatment-related mortality (TRM) and overall survival (OS).

$11 / 72(15 \%)$ of early patients and $7 / 64(11 \%)$ of late patients, and was more prevalent than expected.

The incidence of acute and chronic GVHD was $34 \%$ and $61 \%$, respectively (Table 4). Severe (Grades III-IV) acute GVHD was seen in $11 \%$ of patients. Treatment-related mortality within the first 120 days of transplant was $11 \%$ and within the first year was $21 \%$. Approximately $6 \%$ of patients died of GVHD and $14 \%$ died directly due to infection ( $3 \%$ IFI, $11 \%$ bacterial infection). The 2 -year progressionfree survival and overall survival rates in this high-risk population were $38 \%$ and $47 \%$, respectively (Fig. 2). Disease relapse was the most common cause of death (19 patients; $30 \%)$.

\section{Discussion}

The main focus of this retrospective review was to evaluate the safety and efficacy of VOR as prophylaxis for IFI following alloSCT. Epidemiology studies suggest that there is a

Incidence of acute and chronic graft-versus-host disease

\begin{tabular}{llr}
\hline \multirow{2}{*}{ Timing } & Extent & No. of patients (\%) \\
Acute & Grade I & $8(11 \%)$ \\
& Grades II-IV & $16(23 \%)$ \\
Chronic & Total & $24(34 \%)$ \\
& Limited & $11(17 \%)$ \\
& Extensive & $28(44 \%)$ \\
& Total & $39(61 \%)$ \\
\hline
\end{tabular}

Table 4 
bimodal incidence of IFI following alloSCT $(7,8)$. The initial peak incidence is during marrow aplasia, when neutropenia is severe and barrier breakdown is present (day $<40$ ). Although mold infections predominate, Candida infections also occur. The second peak occurs following day 40 but usually $>$ day 100 with the development of chronic GVHD. Invasive mold and Candida infections occur. The incidence of early IFI has been reported to be approximately $4 \%$ (7). The incidence of late fungal infection is not well reported but generally believed to occur in an additional 10$15 \%$ of patients surviving $>120$ days post alloSCT $(9,10)$.

Our data suggest that antifungal prophylaxis with VOR in the setting of alloSCT is both safe and effective. Only 2 of 72 patients developed early IFI ( 1 while on VOR), an acceptable rate $(2.8 \%)$ of IFI (meets $95 \%$ confidence interval [CI] assuming an expected IFI rate of $4 \%$ [CI 1.88-2.88]). This low incidence of early mold infection confirms 2 prior studies of VOR prophylaxis in alloSCT reported by Siwek et al. (11) and Trifilio et al. (12). Both studies reported a low incidence of mold infections but a higher incidence of breakthrough Candida and zygomycosis infections. The Trifilio study limited VOR prophylaxis to high-risk alloSCT patients (i.e., those taking corticosteroids or with active GVHD). Overall, they reported 6 invasive Candida infections (incidence $10 \%$ ), with Candida glabrata being the most common isolate. Of note, C. glabrata has a high minimum inhibitory concentration for $90 \%$ of organisms ( $\sim 1 \mathrm{mcg} / \mathrm{mL}$ ) with VOR, and several patients in the study had low serum VOR levels. The study used oral VOR dosing of $200 \mathrm{mg}$ b.i.d. and the authors subsequently identified plasma concentrations of VOR $<2 \mathrm{mcg} / \mathrm{mL}$ as a risk factor for IFI, especially Candida. In our study, the mean oral VOR dose was $300 \mathrm{mg}$ b.i.d. It is well established that plasma concentrations are exponentially correlated to dose, thereby an increase from VOR 200 to $300 \mathrm{mg}$ b.i.d. is expected to increase plasma VOR concentrations by $>2.5$-fold. Therefore, it is possible that the weight-based VOR dosing of $4 \mathrm{mg} / \mathrm{kg}$ b.i.d. may have been responsible for the low breakthrough (C. glabrata) infections in this report. These data suggest that measuring and optimizing VOR levels may have therapeutic consequence in preventing breakthrough IFI in immunocompromised alloSCT patients. A prospective trial investigating targeted blood levels and doseadjusted VOR in high-risk alloSCT patients is warranted.

Many studies have raised concern about an increased incidence of zygomycosis infections following use of VOR in alloSCT (13-15). However, the data are still limited, and no conclusions can be made. One theory is that better supportive care, treatments such as VOR, and the use of more potent immunosuppression allow prolonged survival of severely immunocompromised patients, thus giving more time for resistant fungal infections to develop. This retrospective study does not support an increased incidence of zygomycosis. Only a large prospective randomized trial can confirm the association of VOR to zygomycosis infection.

There is no standard antifungal prophylaxis for patients experiencing chronic GVHD (cGVHD) and no published reports on VOR prophylaxis during cGVHD. In this report, a total of 6 of 39 patients developed late definite or probable IFI, but none of these patients developed fungal disease while on VOR. The majority of subjects developed GVHD following tapering of their immunosuppression and after discontinuing VOR. As expected, cGVHD was present before the development of late IFI in most patients, although the initiation of corticosteroids for other reasons (disease relapse, possible autoimmune cytopenia) was an important factor for IFI in 2 patients. A multivariate analysis was performed but failed to identify significant risk factors for late IFI, likely owing to the low incidence of disease. Overall, these data suggest that VOR is effective prophylaxis for preventing late IFI in patients with cGVHD receiving intensive immunosuppression. As mold infections are a significant cause of late morbidity and mortality in those with cGVHD, studies are warranted to better define optimal antifungal therapy during cGVHD (10).

In this cohort of patients, VOR was administered post transplant for a median time of 128 days and was well tolerated. Common side effects of VOR included visual disturbances and liver enzyme elevations. In 3 patients, the abnormal liver tests occurred a mean of 8 days post transplant and this was likely due to preparative therapy toxicity. Three other patients discontinued VOR because of liver test abnormalities a mean of 81 days post transplant and all had possible GVHD. Overall, the side effects fromVOR were not prohibitive and could be managed with dose interruptions and/or dose reductions. VOR administration was strictly prohibited during chemotherapy because of predictable drug-drug interactions. In addition, a $60 \%$ reduction in TAC dosing was required, thus offsetting some of the costs associated with VOR dosing.

In conclusion, VOR prophylaxis in alloSCT is safe and effective at preventing early and late IFI. Evaluating VOR levels and optimizing VOR dosing may help to prevent breakthrough fungal infection. LFTs and the QTc should be monitored in patients receiving VOR as dose adjustments may be required. The low incidence of zygomycosis infections in this study does not support the premise that VOR usage increases the incidence of resistant IFI. Although most centers continue to use FLU as antifungal prophylaxis, VOR usage should be considered in centers where the incidence of mold infection is increased. A randomized clinical trial of FLU versus VOR prophylaxis in alloSCT is ongoing and should further clarify the role of VOR as prophylaxis (http://clinicaltrials.gov/ct/show/NCT00023530? 
order=1). A randomized trial of VOR versus posaconazole prophylaxis in alloSCT would also be of value.

\section{Acknowledgement:}

This manuscript was supported by an unrestricted research grant from Pfizer Inc.

\section{References}

1. Goodman JL, Winston DJ, Greenfield RA, et al. A controlled trial of fluconazole to prevent fungal infections in patients undergoing bone marrow transplantation. N Engl J Med 1992; 326 (13): 845-851.

2. Marr KA, Seidel K, Slavin MA, et al. Prolonged fluconazole prophylaxis is associated with persistent protection against candidiasis-related death in allogeneic marrow transplant recipients: long-term follow-up of a randomized, placebo-controlled trial. Blood 2000; 96 (6): 2055-2061.

3. Slavin MA, Osborne B, Adams R, et al. Efficacy and safety of fluconazole prophylaxis for fungal infections after marrow transplantation - a prospective, randomized, double-blind study. J Infect Dis 1995; 171 (6): 1545-1552.

4. Ullmann A, Lipton J, Vesole D, et al. Posaconazole or fluconazole for prophylaxis in severe graft-versus-host disease. N Engl J Med 2007; 356 (4): 335-347.

5. Herbrecht R, Denning DW, Patterson TF, et al.Voriconazole versus amphotericin B for primary therapy of invasive aspergillosis. N Engl J Med 2002; 347 (6): 408-415.

6. Ascioglu S, Rex JH, de Pauw B, et al. Defining opportunistic invasive fungal infections in immunocompromised patients with cancer and hematopoietic stem cell transplants: an international consensus. Clin Infect Dis 2002; 34 (1): 7-14.

7. Bhatti Z, Shaukat A, Almyroudis NG, Segal BH. Review of epidemiology, diagnosis, and treatment of invasive mould infections in allogeneic hematopoietic stem cell transplant recipients. Mycopathologia 2006; 162 (1): 1-15.

8. Wald A, Leisenring W, van Burik JA, Bowden RA. Epidemiology of aspergillus infections in a large cohort of patients undergoing bone marrow transplantation. J Infect Dis 1997; 75 (6): 1459-1466.

9. Fukuda T, Hackman RC, Guthrie KA, et al. Risks and outcomes of idiopathic pneumonia syndrome after nonmyeloablative and conventional conditioning regimens for allogeneic hematopoietic stem cell transplantation. Blood 2003; 102 (8): 2777-2785.

10. Marr KA, Carter RA, Boeckh M, Martin P, Corey L. Invasive aspergillosis in allogeneic stem cell transplant recipients: changes in epidemiology and risk factors. Blood 2002; 100 (13): 4358-4366.

11. Siwek GT, Pfaller MA, Polgreen PM, et al. Incidence of invasive aspergillosis among allogeneic hematopoietic stem cell transplant patients receiving voriconazole prophylaxis. Diagn Microbiol Infect Dis 2006; 55 (3): 209-212.

12. Trifilio S, Singhal S, Williams S, et al. Breakthrough fungal infections after allogeneic hematopoietic stem cell transplantation in patients on prophylactic voriconazole. Bone Marrow Transplant 2007; 40 (5): 451-456.

13. Marty FM, Cosimi LA, Baden LR. Breakthrough zygomycosis after voriconazole treatment in recipients of hematopoietic stem-cell transplants. N Engl J Med 2004; 350 (9): 950-952.

14. Siwek GT, Dodgson KJ, de Magalhaes-Silverman M, et al. Invasive zygomycosis in hematopoietic stem cell transplant recipients receiving voriconazole prophylaxis. Clin Infect Dis 2004; 39 (4): 584-587.

15. Trifilio SM, Bennett CL, Yarnold PR, et al. Breakthrough zygomycosis after voriconazole administration among patients with hematologic malignancies who receive hematopoietic stem-cell transplants or intensive chemotherapy. Bone Marrow Transplant 2007; 39 (7): 425-429. 